\title{
Рекомбинация носителей заряда через возбужденные уровни бора в кремнии при низких температурах
}

\author{
(C) T.T. Муратов \\ Ташкентский государственный педагогический университет им. Низами, \\ 100185 Ташкент, Узбекистан \\ E-mail: temur-muratov@yandex.ru
}

Поступила в Редакцию 11 февраля 2019 г.

В окончательной редакции 2 августа 2019 г.

Принята к публикации 7 августа 2019 г.

\begin{abstract}
Изучен процесс рекомбинации носителей через мелкие примесные центры бора в легированном умеренно компенсированном кремнии. Основное внимание было уделено теоретическому объяснению „эмпирических“ температурных зависимостей времени жизни $\tau(T)$ носителей в интервале температур (1.7-4.2) K при концентрациях легирующей примеси $n_{\mathrm{B}} \geq 10^{14} \mathrm{~cm}^{-3}$ с компенсацией $\leq 10 \%\left(n_{d}+n_{a} \leq 10^{13} \mathrm{~cm}^{-3}\right)$. Довольно точно удалось установить, что мелкий возбужденный уровень с энергией связи 5 мэВ ( $3 s$-состояние) является квазирезонансным. Получены приближенные формулы для коэффициента захвата. Изучается влияние „слабого“ магнитного поля (10-100)Гс на величину коэффициента захвата; при этом показано, что „слабое“ магнитного поле слегка уменьшает время жизни носителей, тем самым стимулируя процесс их рекомбинации.
\end{abstract}

Ключевые слова: маршруты рекомбинации, резонансный уровень, коэффициент захвата, время жизни, фотопроводимость, классические „слабое“ и „сильное“ магнитные поля.

DOI: $10.21883 /$ FTP.2019.12.48611.9078

\section{1. Введение}

Примеси бора в кремнии создают акцепторные уровни, отстоящие от потолка валентной зоны на расстоянии 0.045 эВ (уровень $E_{B}=E_{V}+0.045$ эВ), что примерно в 25 раз меньше ширины запрещенной зоны матрицы $\mathrm{Si}$ (1.15 эВ). Следовательно, возбужденные уровни примеси локализованы вблизи валентной зоны. При определенных условиях такие уровни (так называемые $A^{0}$-центры) могут проявлять себя в качестве рекомбинационных центров [1]. Теоретически возможны различные маршруты рекомбинации носителей заряда: прямые переходы из зоны в зону - непосредственная рекомбинация электрона и дырки (рис. 1, переход 1); рекомбинация через примесные центры - дырка из валентной зоны $(V$-зоны $)$, сначала захватывается мелким примесным уровнем ( $A^{0}$-центром), а затем уже этим же уровнем захватывается электрон из зоны проводимости $(C$-зоны) (другими словами, электрон, захваченный $A^{+}$-центром, по истечении определенного промежутка времени сваливается в $V$-зону) (рис. 1, переходы 4-6). Этот процесс, разумеется, может идти и в обратном порядке. В равной степени это относится и к $D^{-}$-центрам.

В том случае, если $A^{+}$-центры размываются в одну примесную $A^{+}$-зону (при концентрациях бора $n_{\mathrm{B}}=(1-7) \cdot 10^{16} \mathrm{~cm}^{-3}$ и температуре $\left.T \approx 18 \mathrm{~K}\right)$, процесс рекомбинации заключается в том, что термолизованный электрон сначала движется по $A^{+}$-зоне с последующим переходом на $V$-зону [2]. Однако такой канал рекомбинации требует предварительного „остывания“ электрона, путем испускания им „мелких“ фононов [3].
При высоких температурах (порядка комнатных и выше) рекомбинация может происходить через глубокие центры [4] (рис. 1, переходы 2-3). Рекомбинация посредством прыжкового механизма проводимости при наличии слабого магнитного поля была рассмотрена в работе [5]. Принцип „детального равновесия“ также допускает захват дырок нейтральным акцептором

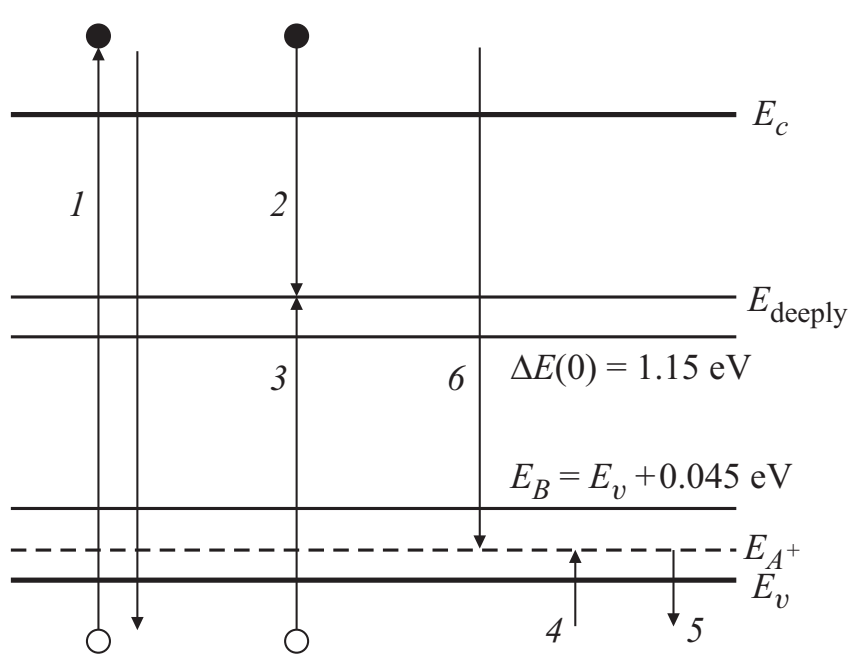

Рис. 1. Маршруты возбуждения и рекомбинации: $1-$ межзонное возбуждение носителей заряда, 2, 3 - захват электронов и дырок глубокими центрами, 4, 5 - захват дырок нейтральным бором и их реэмиссия, $6-$ захват электронов $A^{+}$-центрами. В представленной модели $E_{A}^{+}$соответствует энергии связи дырки с $A^{0}$-центром $\left(E_{A}^{+} \approx 5\right.$ мэВ). Следует понимать условность электронно-дырочных переходов. 
$\left(A^{0} \pm p^{+} \rightarrow A^{0}\right)$, с последующей их реэмиссией (рис. 1 , переходы 4-5).

Атом бора в невозбужденном состоянии имеет электронную конфигурацию $1 s^{2} 2 s^{2} 2 p^{1}$. Энергия ионизации мелкого акцептора 0.045 эВ. Согласно экспериментальным данным [1], энергия связи дырки (электрона) с нейтральным атомом бора оказалась равной 5 мэВ. Учитывая водородоподобную структуру мелкой примеси $\mathrm{Si}: \mathrm{B}$, нетрудно понять, что дополнительный электрон захватывается на третий уровень атома бора $(n=3)$, с образованием короткоживущего отрицательного иона с конфигурацией $1 s^{2} 2 s^{2} 2 p^{1} 3 s^{1}$. Радиус локализации дополнительного электрона $r_{3}^{*}=9 \chi \hbar^{2} /\left(m^{*} e^{2}\right) \approx 105 \AA(\chi \approx 11.7-$ диэлектрическая постоянная, $m^{*} \approx 0.5 m_{0}-$ эффективная масса электрона в кремнии, $e=4.8 \cdot 10^{-10}$ СГС), что сопоставимо с его длиной волны $\lambda \approx \hbar / \sqrt{3 m^{*} k_{\mathrm{B}} T} \approx 140 \AA$ при температуре $T \approx 3 \mathrm{~K}$ ( $k_{\mathrm{B}}$ - постоянная Больцмана). Следовательно, захват дополнительного электрона на третий уровень имеет резонансный характер. Возникает вопрос - почему дополнительный электрон не был захвачен $p$-орбитой, в соответствии с правилом Клечковского заполнения электронных оболочек. Дело в том, что энергия захвата (электронного сродства) 5 мэВ соответствует типичным энергиям невырожденных носителей заряда при температурах $\sim(10-100) \mathrm{K}$, и в этом смысле третий уровень $E_{3}=\varepsilon$ является мелким уровнем захвата дополнительного электрона. ${ }^{1}$

С понижением температуры и увеличением уровня фотовозбуждения концентрация $A^{+}$-центров растет, и примеси бора начинают оказывать существенное влияние на процесс рекомбинации носителей.

Расчеты, проведенные в работе [1], дают зависимости времени жизни $\tau_{n}$ электрона и $\tau_{p}$ дырки от температуры и уровня подсветки, близкие к экспериментальным значениям, если положить коэффициент захвата электрона $A^{+}$-центром $\alpha_{n}=5 \cdot 10^{-6} \mathrm{~cm}^{3} / \mathrm{c}$ и энергию связи дырки с нейтральным бором $\varepsilon=5$ мэВ. Однако при этом не устанавливается связь коэффициента захвата с энергией связи носителя и температурой: изменение одного параметра, например температуры, непременно приводит к изменению другого: $\alpha_{n}$ и(или) $\varepsilon$.

В связи с этим установление теоретической формулы для $\alpha_{n}$, охватывающей актуальный интервал (1.7-4.2) K, является принципиальной задачей. Тот факт, что после подстановки значений $\alpha_{n}=5 \cdot 10^{-6} \mathrm{~cm}^{3} /$ с и $\varepsilon=5$ мэВ в формулу $\tau_{n}=1 /\left(\alpha_{n} n_{\mathrm{B}}\right) \quad\left(n_{\mathrm{B}} \approx 1.5 \cdot 10^{14} \mathrm{~cm}^{-3}-\right.$ концентрация $A^{+}$-центров) получаются зависимости, соответствующие экспериментальным данным [1], явля-

\footnotetext{
${ }^{1}$ Согласно распределению Максвелла нет необходимости, чтобы энергия носителей заряда была близка к $\varepsilon$. Иными словами, на $s$-уровень (5 мэВ) наиболее эффективно захватываются носители, соответствующие интервалу температур (10-100) К. Однако это не исключает возможности резонансного захвата носителей из интервала $(1-10) \mathrm{K}[1]$. В этом смысле кремний, легированный бором, редкое исключение, где резонансный захват носителей может (в принципе) доминировать в широкой области температур $(1-100) \mathrm{K}$.
}

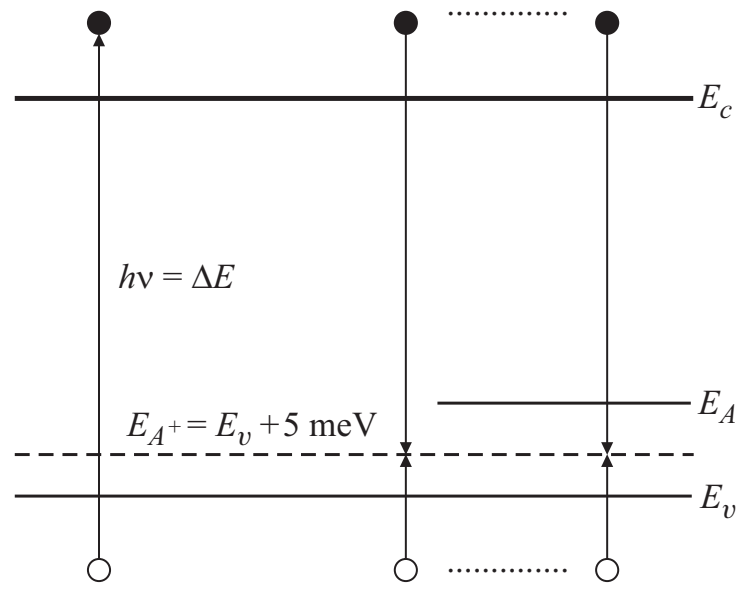

Рис. 2. Свободные носители заряда (электроны, дырки) непрерывно генерируются под действием внешнего источника фотовозбуждения. Рекомбинация происходит благодаря резонансному захвату дырки $A^{0}$-центром с последующим захватом электрона: $p^{+}+A^{0} \rightarrow A^{+}+e^{-} \rightarrow A^{0}$.

ется неубедительным, без соответствующего рассмотрения теоретической зависимости $\alpha_{n}(\varepsilon, T)$. Принципиальным достоинством работы [1] следует считать идею о рекомбинации носителей заряда через мелкие уровни прилипания. Вывод теоретической формулы для коэффициента захвата должен быть основан на модели, адекватной рассматриваемой ситуации. В качестве объекта исследования можно взять модельный полупроводник с малой концентрацией носителей $\left(n_{e} \geq 10^{10} \mathrm{~cm}^{-3}\right)$ при очень низких температурах $(\leq 10 \mathrm{~K})$. Этим исключаются межзонная излучательная, экситонная и Оже рекомбинации (ударная), а также рекомбинация через глубокие уровни (рис. 1, переходы 2-3). При таких условиях эффекты, связанные с резонансным захватом носителей, наиболее ярко проявляются и их можно четко идентифицировать в эксперименте [6-8].

Таким образом, модель, представленную на рис. 1, следует упростить (рис. 2).

Цель настоящей работы - установление теоретического соотношения (аналитических оценок) для коэффициента захвата (вероятности захвата), адекватно соответствующего актуальному для эксперимента интервалу температур. Рассматривается вопрос о влиянии „слабого“ магнитного поля на величину коэффициента захвата. Анализируется стационарная фотопроводимость.

\section{2. Методика расчета}

Наличие мелкого $3 s$-уровня, образованного примесным потенциалом бора и ответственного за резонансный захват носителей, можно учесть в приближении $s$-рассеяния, в рамках которого выражение для сечения 
рассеяния (захвата) имеет наиболее простой вид: ${ }^{2}$

$$
\sigma=\frac{2 \pi \hbar^{2}}{m^{*}} \frac{1}{E+\varepsilon}(\varepsilon>0), \quad \sigma \approx 40 \pi a_{\mathrm{B}}^{2}\left(a_{\mathrm{B}} \approx 12 \AA\right),
$$

где $E$ - энергия рассеиваемого электрона (дырки), $a_{\mathrm{B}}-$ боровский радиус мелкой примеси.

На основе формулы (1) вычисляем коэффициент захвата:

$$
\begin{aligned}
\alpha_{n}=\langle v \sigma\rangle_{E}=\sqrt{\frac{2}{m^{*}}} \frac{2 \pi \hbar^{2}}{m^{*}} \frac{\int_{0}^{\infty} E \frac{\sqrt{E}}{E+\varepsilon} \exp \left(-\frac{E}{k_{\mathrm{B}} T}\right) \sqrt{E} d E}{\int_{0}^{\infty} E \exp \left(-\frac{E}{k_{\mathrm{B}} T}\right) \sqrt{E} d E} \\
=\sqrt{\frac{2}{\pi m^{*}}} \frac{8 \pi \hbar^{2}}{3 m^{*}} \frac{1}{\sqrt{k_{\mathrm{B}} T}} \int_{0}^{\infty} \frac{x^{2} \exp (-x)}{x+x_{0}} d x \\
=\sqrt{\frac{2}{\pi m^{*}}} \frac{8 \pi \hbar^{2}}{3 m^{*}} \frac{1}{\sqrt{k_{\mathrm{B}} T}}\left[1-x_{0} L\left(x_{0}\right)\right] .
\end{aligned}
$$

При этом $L\left(x_{0}\right)=1-x_{0} \exp \left(x_{0}\right)\left[-\operatorname{Ei}\left(-x_{0}\right)\right]$, $x_{0}=\varepsilon / k_{\mathrm{B}} T, v=\sqrt{2 E / m^{*}},-\operatorname{Ei}\left(-x_{0}\right)-$ интегральный логарифм.

Потребность в точной формуле (2) отпадает, если учесть то обстоятельство, что энергии связи электрона в 5 мэВ соответствует температура $58 \mathrm{~K}$, которая гораздо больше чем $3 \mathrm{~K}$.

Следовательно, $\varepsilon \gg k_{\mathrm{B}} T$, и

$$
\begin{gathered}
L\left(x_{0}\right)=1-x_{0} \exp \left(x_{0}\right)\left[-\operatorname{Ei}\left(-x_{0}\right)\right] \approx 1 / x_{0}-2 / x_{0}^{2}+\ldots \\
\left(x_{0} \gg 1\right) .
\end{gathered}
$$

Подставляя разложение (3) в (2), получим

$$
\alpha_{n} \approx \sqrt{\frac{2 \pi}{m^{*}}} \frac{16 \hbar^{2}}{3 m^{*}} \frac{1}{\sqrt{k_{\mathrm{B}} T}} \frac{k_{\mathrm{B}} T}{\varepsilon} \propto T^{1 / 2} .
$$

Если подставить в формулу (4) значения $m^{*}=0.5 m_{0}$, $\varepsilon=5$ мэВ и $T=3 \mathrm{~K}$, получим $\alpha_{n}=4 \cdot 10^{-6} \mathrm{~cm}^{3} / \mathrm{c}$. Видно, насколько близким оказалось теоретическое значение коэффициента захвата с его экспериментально измеренным значением $\alpha_{n}=5 \cdot 10^{-6} \mathrm{~cm}^{3} / \mathrm{c}$ [1]. Теоретическая формула (4) приводит к зависимости $\tau_{n} \sim T^{-1 / 2}$, которая качественно достраивает нижнюю часть эмпирической (степенной) зависимости $\tau_{n} \sim T^{4}-T^{5}$ для концентрации бора $n_{\mathrm{B}} \geq 10^{14} \mathrm{~cm}^{-3}$ в интервале $(1.7-4.2) \mathrm{K}$ [1] (рис. 3). Однако существенно расходится с зависимостью $\tau_{n} \sim T-T^{3 / 2}$ для концентрации $n_{\mathrm{B}} \leq 10^{13} \mathrm{~cm}^{-3}$ $\left(K=n_{d} / n_{a}=10^{-1}\right)$ в указанном интервале температур. Расхождение теории с экспериментом в области $n_{\mathrm{B}} \leq 10^{13} \mathrm{~cm}^{-3}$ разрешается, если учесть, что функция

\footnotetext{
${ }^{2}$ Тепловое размытие $E_{3}=\varepsilon$ уровня примерно на два порядка меньше среднего расстояния между кулоновскими уровнями: $\Delta E_{\text {ср }} \approx 3.3$ мэВ, $\Delta E_{T} \approx h / \tau_{T} \approx 0.066$ мэВ. Здесь $\tau_{T}$ - время тепловой релаксации электронов $\left(\tau_{T} \approx 10^{-11} \mathrm{c}\right)$. Правомерность приближения $s$-рассеяния очевидна.
}

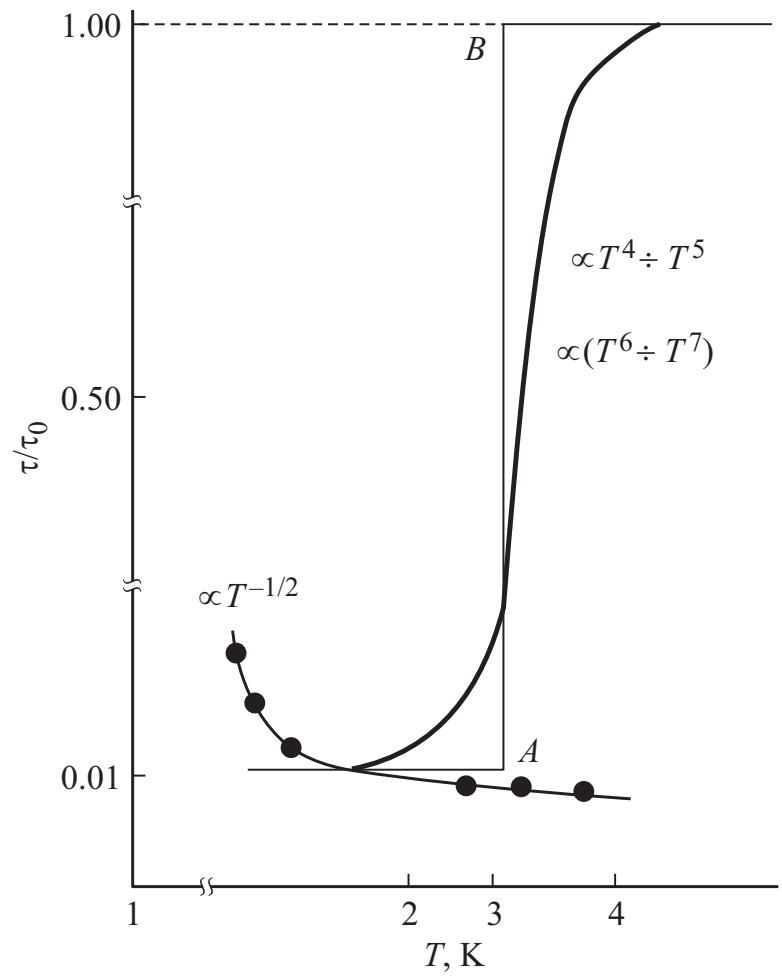

Рис. 3. Идеализированная картина резкого спада времени жизни носителей в интервале $(1.7-4.2) \mathrm{K}$. Столь резкий спад, более чем на два порядка, может быть обусловлен только резонансным захватом носителя на $D^{-}\left(A^{+}\right)$-центр. Теоретическая зависимость $T^{-1 / 2}$ (кружки) качественно передает нижнюю часть спада. Другими словами, время жизни носителей соответствует асимптотике их времени релаксации при резонансном рассеянии $T^{-1 / 2}$. Влияние „слабого“ магнитного поля сводится к уменьшению времени жизни и в пределе характеризуется вертикальной линией $A B$ в окрестности точки $3 \mathrm{~K}$. Верхняя часть графика соответствует не зависящему от температуры времени жизни (область примесной проводимости).

$L\left(x_{0}\right)$ наряду с разложением (3) допускает также асимптотику

$$
L\left(x_{0}\right)=1-x_{0} \exp \left(x_{0}\right)\left[-\operatorname{Ei}\left(-x_{0}\right)\right] \rightarrow 0,
$$

с тем, что

$$
-\operatorname{Ei}\left(-x_{0}\right)=\int_{x_{0}}^{\infty} \frac{\exp (-t)}{t} d t \approx \frac{\exp \left(-x_{0}\right)}{x_{0}} \quad \text { при } \quad x_{0}>5 .
$$

На основе (5) получаем теоретическую зависимость $\tau_{n} \sim T^{1 / 2}$, которая достаточно хорошо соответствует экспериментальной зависимости $\tau_{n} \sim T-T^{3 / 2}[1]$. В данном случае $\alpha_{n}$ очень слабо зависит от энергии связи $\varepsilon$. Этим объясняется „стабильность“ времени жизни $\tau_{n, p}$ носителей к вариациям интенсивности фотовозбуждения [1]. Можно утверждать, что при концентрациях примеси $n_{\mathrm{B}} \leq 10^{13} \mathrm{~cm}^{-3}$ и температурах $T \leq 12 \mathrm{~K}$, носители сла- 
бо резонируют на мелких уровнях захвата, и в основном они рассеиваются на ионах примеси бора. ${ }^{3}$

Таким образом, при значениях $n_{\mathrm{B}} \leq 10^{13} \mathrm{~cm}^{-3}$ и $T \leq 12 \mathrm{~K}$ резонансный захват носителей в значительной степени перекрывается их обычным кулоновским рассеянием на ионах примеси. Оценка времени жизни $\tau_{n}=1 /\left(\alpha_{n} n_{\mathrm{B}}\right)$ неравновесных электронов дает $\tau_{n} \sim(1-10)$ нс.

В среднем время жизни электронов $\tau_{n}$ на порядок превышает время жизни дырок $\tau_{p}\left(\tau_{p} / \tau_{n} \sim T^{2}\right)[1]$. Вообще, наличие резкого спада времени жизни $\tau_{n} \sim T^{4}-T^{5}$ (для электронов) и $\tau_{p} \sim T^{6}-T^{7}$ (для дырок) в небольшом интервале температур (1.7-4.2) K уже указывает на резонансный характер захвата носителей на $D^{-}\left(A^{+}\right)$центры (рис. 3).

Стационарная фотопроводимость $\Delta \sigma_{\mathrm{st}}=e G \mu \tau_{n}=$ $=\mathrm{const} / T$ (при $\tau_{n} \sim T^{-1 / 2}$ ) слабо зависит от температуры $\left(\mu \sim T^{-1 / 2}-\right.$ подвижность электрона при резонансном рассеянии [8], $G$ - скорость фотовозбуждения). Так что она не вносит существенного вклада в увеличение электропроводности.

\section{3. Влияние магнитного поля на кинетику рекомбинации неравновесных носителей}

Исследованию влияния „сильного“ магнитного поля на кинетику рекомбинации в полупроводниках $(\mathrm{Ge})$ была посвящена работа [7]. Согласно результатам расчетов, при значениях магнитного поля $H \geq 2.2$ кГс время жизни неравновесных носителей в $\mathrm{Ge}$ увеличивается более чем в 100 раз. В то же время, вопрос о влиянии „слабого“ магнитного поля на кинетику рекомбинации остается невыясненным. Следует подчеркнуть, что понятие „сильного“ и „слабого“ магнитного поля имеет условный характер. Так, для слабо легированного германия подвижность электронов составляет при $300 \mathrm{~K}$ $\mu \approx 3 \cdot 10^{3} \mathrm{~cm}^{2} /$ В $\cdot$ с. Поле $H \geq 2.2$ кГс будет приближенно удовлетворять критерию $\mu \cdot H / c \ll 1(c-$ скорость света). При $10 \mathrm{~K}$ подвижность электронов того же германия будет $\mu \geq 10^{4} \mathrm{~cm}^{2} / \mathrm{B} \cdot \mathrm{c}$, и то же самое магнитное поле в $H \geq 2.2$ кГс будет сильным [7]. Ясно, что поля $\sim 100$ Гс будут слабыми в интервале $T=(1.7-4.2) \mathrm{K}$. Теоретически вопрос упирается в поиск подходящего приближения для учета влияния „слабого“ магнитного поля на коэффициент захвата. Искомое приближение не может быть основано на „кружковом“ приближении [7]. Правильный подход состоит в том, чтобы рассматривать „слабое“ магнитное поле как возмущение, а именно учесть влияние магнитного поля как локальное „растяжение“ поперечника $\left(\sigma_{T}=\pi r_{T}^{2}\right) A^{0}$-центра (рис. 4): $r_{T} \approx \lambda, \varepsilon \approx k_{\mathrm{B}} T, \Delta \sigma_{T}=2 \pi r_{T} \Delta r, \Delta r / \Delta l \approx r_{T} / l_{0}$. Откуда $\Delta r \approx\left(r_{T} / l_{0}\right) \Delta l \approx\left(\varphi^{2} / 2\right) r_{T}$.

\footnotetext{
3 Теперь становится понятным происхождение вклада $T^{3 / 2}$ в экспериментальной зависимости $\tau_{n} \sim T-T^{3 / 2}$.
}

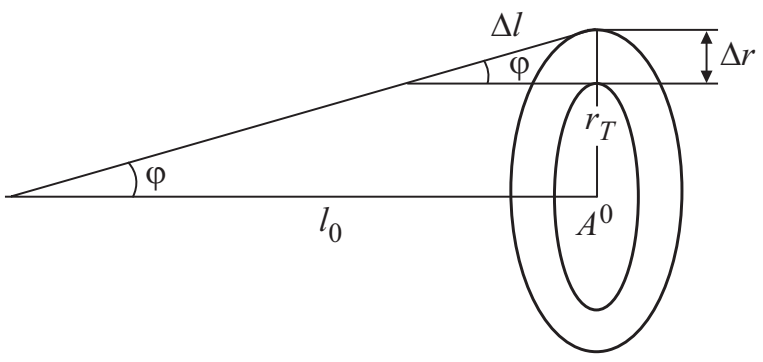

Рис. 4. „Рассеивающее“ влияние слабого магнитного поля рассматривается как локальное растяжение эффективного „поперечника" $A^{0}$-центра. Здесь $\varphi-$ угол отклонения носителя от первоначального направления движения, $l_{0}$ - средняя длина свободного пробега носителя при $H=0, \Delta l$ - укорочение $l_{0}$, $\Delta r$ - локальное растяжение эффективного радиуса $A^{0}$-центра, $r_{T}$ - эффективный „поперечник“ $A^{0}$-центра, $r_{H}=r_{T}+\Delta r$.

Понятно, что

$$
\sigma_{H} \approx \sigma_{T}\left(1+\frac{\pi}{4} \frac{\bar{E}_{H}}{k_{\mathrm{B}} T}\right) .
$$

Здесь $\sigma_{H}$ - эффективное сечение захвата при наличии слабого магнитного поля,

$$
\bar{E}_{H}=\frac{m^{*}\left(\omega_{H} l_{0}\right)^{2}}{2}
$$

- средняя кинетическая энергия носителя на малой части циклотронной орбиты $\left(\bar{E}_{H} \propto H^{2}\right), \omega_{H}$ - циклотронная частота.

На основе выражения для $\sigma_{H}$ и формулы (2) нетрудно понять, что

$$
\alpha_{H}(T) \approx \alpha_{T}\left(1+\frac{\pi}{4} \frac{\bar{E}_{H}}{k_{\mathrm{B}} T}\right)
$$

где $\alpha_{T}$ определяется выражением (4).

При выводе возмущающего члена (диамагнитной поправки) $\frac{\pi}{4} \frac{\bar{E}_{H}}{k_{\mathrm{B}} T}$ было учтено, что при резонансном рассеянии ${ }^{4}$

и

$$
\mu \approx \frac{2 e}{3 \pi \hbar^{2} n_{\mathrm{B}}} \sqrt{\frac{m^{*}}{2 \pi}} \frac{\varepsilon}{\sqrt{k_{\mathrm{B}} T}}
$$

$$
l_{0} \approx \frac{2 m^{*} \varepsilon}{\pi \hbar^{2} n_{\mathrm{B}}} \approx 2 \cdot 10^{-3} \mathrm{cM} \quad(\varphi=\mu H / c \ll 1) .
$$

Анализируя полученный результат (6), можно сказать, что в пределе $H \rightarrow 0$ магнитное поле слегка уменьшает время жизни неравновесных носителей (рис. 3). В этом смысле результат (6) резко отличается от результата $\alpha_{H} / \alpha_{T}=10^{-2}$ [7] (см. Приложсение). Таким образом, „слабое“ магнитное поле несколько ускоряет динамику

\footnotetext{
4 Формулы для подвижности и длины свободного пробега в условиях резонансного захвата получаются на основе формулы (1) [9] При значениях $H=\left(10^{2}-10^{3}\right)$ Гс поправочный член в (6) порядка $\left(10^{-3}-10^{-2}\right)$. В области $H>10^{3}$ Гс формула (6) неприменима и должна быть заменена „кружковым“ приближением [7].
} 
захвата. Тот факт, что результат $\alpha_{H} / \alpha_{T}=10^{-2}$ получен в условиях каскадного захвата носителей, не имеет принципиального значения. В любом случае высоковозбужденный фотоэлектрон термолизуется до $\varepsilon=5$ мэВ, релаксируя свою энергию на испускание тепловых фононов [10].

\section{4. Заключение}

Основное содержание настоящей работы можно резюмировать формулами (4) и (6). На основе формулы (4) получено теоретическое значение коэффициента захвата $\alpha_{n}=4 \cdot 10^{-6} \mathrm{~cm}^{3} / \mathrm{c}$, которое близко сошлось с его экспериментальным значением $5 \cdot 10^{-6} \mathrm{~cm}^{3} / \mathrm{c}$ в интервале температур (1.7-4.2) K [1]. Это позволяет заключить, что в указанном интервале температур при концентрациях легирующей примеси $n_{\mathrm{B}} \geq 10^{14} \mathrm{~cm}^{-3}$ захват носителей на мелкий уровень бора носит резонансный характер. При концентрациях $n_{\mathrm{B}} \leq 10^{13} \mathrm{~cm}^{-3}(K=0.1)$ резонансный захват выражен очень слабо. Качественно интерпретировано происхождение эмпирических зависимостей $\tau_{n} \sim T^{4}-T^{5}$ и $\tau_{n} \sim T-T^{3 / 2}$. В частности, теоретическая зависимость $\tau_{n} \sim T^{1 / 2}$ соответствует $\tau_{n} \sim T-T^{3 / 2}$ области $T \leq 12 \mathrm{~K}$, где носители преимущественно рассеиваются на ионах примеси бора. Теоретическая зависимость $\tau_{n} \sim T^{-1 / 2}$ качественно довершает нижнюю часть быстрого спада $\tau_{n} \sim T^{4}-T^{5}\left(\tau_{p} \sim T^{6}-T^{7}\right)$ в интервале $(1.7-4.2) \mathrm{K}$ (рис. 3). Теоретический интерес представляет именно нижняя часть спада, так как она замыкает крутой спад и сменяет его подъемом. Тем самым, зависимости $\tau_{n} \sim T^{4}-T^{5}\left(\tau_{p} \sim T^{6}-T^{7}\right)$ играют роль своего рода связующего звена между теорией и экспериментом. Их основное предназначение - имитация резкого спада времени жизни в условиях резонансного захвата.

\section{Приложение}

На основе (6) получаются предельные формулы:

$$
\begin{gathered}
\alpha_{H}^{\max }=\frac{32}{3} \frac{\omega_{H}}{n_{\mathrm{B}}}, \quad \tau_{\min }=\frac{3}{32 \omega_{H}}, \\
\left(\alpha_{H} \leq \alpha_{H}^{\max }\right), \quad\left(\tau_{H} \geq \tau_{\min }\right) .
\end{gathered}
$$

Для оценки порядка величин положим $H=100$ Гс, в этом случае $\omega_{H} \approx 4 \cdot 10^{9}$ рад $/ \mathrm{c}$. Тогда $\alpha_{H}^{\max } \approx 4 \cdot 10^{-4} \mathrm{~cm}^{3} / \mathrm{c}$ и $\tau_{\min } \approx 2.7 \cdot 10^{-11} \mathrm{c}\left(\tau_{\min } \geq \tau_{T}\right)$, откуда

$$
\frac{\alpha_{H}^{\max }}{\alpha_{T}}=\frac{4 \cdot 10^{-4}}{4 \cdot 10^{-6}}=10^{2} .
$$

Далее с учетом (4) для актуального интервала $(1.7-4.2) \mathrm{K}\left(n_{\mathrm{B}}=10^{14} \mathrm{~cm}^{-3}\right)$

$$
\bar{\tau}=\frac{1}{T_{2}-T_{1}} \int_{T_{1}}^{T_{2}} \tau(T) d T \approx 2.7 \cdot 10^{-9} \mathrm{c} .
$$

\section{Конфликт интересов}

Автор заявляет об отсутствии конфликта интересов.

\section{Список литературы}

[1] Е.М. Гершензон, Ю.П. Ладыжинский, А.П. Мельников. Письма ЖЭТФ, 14 (9), 380 (1971).

[2] В.В. Рыльков. ФТП, 22 (9), 1661 (1988).

[3] Е.И. Воеводин, Е.М. Гершензон, Г.Н. Гольцман, Н.Г. Птицина, Г.М. Чулкова. ФТП, 22 (3), 540 (1988).

[4] Э.З. Имамов, О.В. Курносова, А.А. Пахомов. ФТТ, 31 (3), 211 (1989).

[5] А.П. Мельников, Ю.А. Гурвич, Л.Н. Шестаков, Е.М. Гершензон. Письма ЖЭТФ, 73 (1), 50 (2001).

[6] Е.М. Гершензон, А.И. Мельников, Р.И. Рабинович, Н.А. Серебрякова. УФН, 132 (2), 353 (1980).

[7] Б.Б. Зеленер, Б.В. Зеленер, Э.А. Маныкин. Письма ЖЭТФ, 95 (3), 164 (2012).

[8] Э.З. Имамов, Н.М. Колчанова, Л.Н. Крещук, И.Н. Яссиевич. ФТТ, 27 (1), 69 (1985).

[9] С.Г. Дмитриев, В.В. Рыльков, О.Г. Шагимуратов. ФТП, $25(2), 360$ (1991).

[10] В.Ф. Банная, Е.В. Никитина. ФТП, 53 (1), 13 (2019).

Редактор Г.А. Оганесян

\section{Recombination of charge carriers across boron exited levels in silicon at low temperatures}

\section{T.T. Muratov}

\section{Tashkent State Pedagogical University \\ named after Nizami, \\ 100185 Tashkent, Uzbekistan}

\begin{abstract}
In present article was studied the process of recombination of charge carriers across shallow impurity boron centers in silicon at low temperatures. Primary emphasis was received by theoretical interpretation of empiric temperature dependencies of lifetime $\tau(T)$ of the carriers in the temperature range of $(1.7-4.2) \mathrm{K}$ under doped impurity concentrations $n_{\mathrm{B}} \geq 10^{14} \mathrm{sm}^{-3}$ with compensation $\leq 10 \%\left(n_{d}+n_{a} \leq 10^{13} \mathrm{sm}^{-3}\right)$. It is enough exactly determined that shallow excited level with binding energy $5 \mathrm{meV}(3 s$-state) is almost resonant. The approximate formulas for the coefficient of resonant capture are obtained. Influence of weak magnetic field $(10-100)$ Gs on value of the coefficient of resonant capture is analyzed, as for showed that weak magnetic field (in order $10 \mathrm{Gs}$ ) loosely decreases of carriers lifetime in such a way to stimulate a process of their recombination.
\end{abstract}

